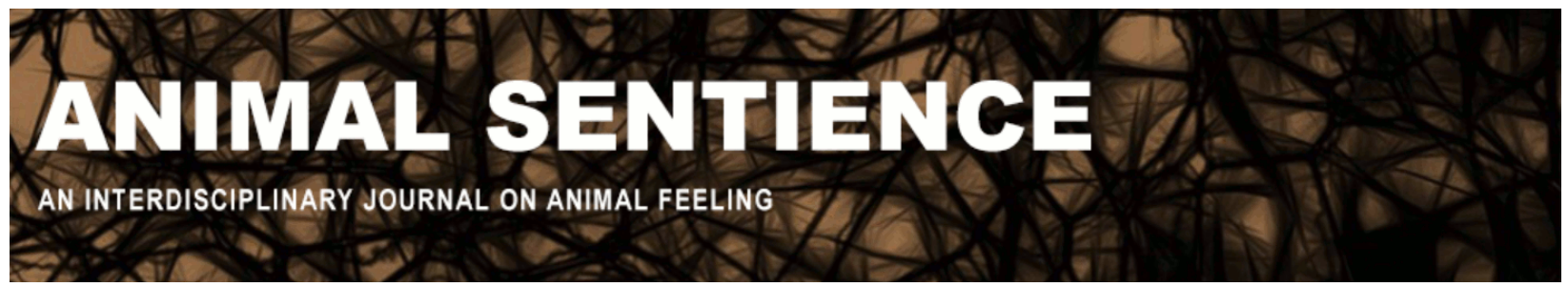

Adolphs, Ralph (2017) Operationalizing fear through understanding vigilance. Animal Sentience 15(2)

DOI: $10.51291 / 2377-7478.1214$

Date of submission: 2017-10-03

Date of acceptance: 2017-10-10

(c)

This article has appeared in the journal Animal

Sentience, a peer-reviewed journal on animal

cognition and feeling. It has been made open access,

free for all, by WellBeing International and deposited

in the WBI Studies Repository. For more information,

please contact

wbisr-info@wellbeingintl.org.

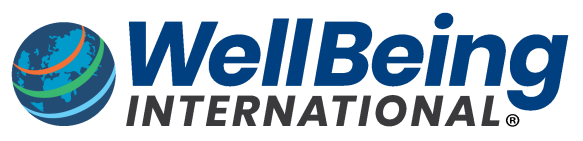

SOLUTIONS FOR PEOPLE, ANIMALS AND ENVIRONMENT 


\title{
Operationalizing fear through understanding vigilance
}

Commentary on Beauchamp on Fear \& Vigilance

\author{
Ralph Adolphs \\ California Institute of Technology
}

\begin{abstract}
Beauchamp's target article raises important questions about the features that often accompany fear. How reliable an indicator of fear is vigilance? Is it constitutive, cause, or consequence of fear? These questions force us towards a clearer definition of "fear."
\end{abstract}

\begin{abstract}
Ralph Adolphs is a social neuroscientist who studies emotions in humans. Bren Professor of Psychology, Neuroscience, and Biology at California Institute of Technology, Allen V. C. Davis and Lenabelle Davis Leadership Chair and Director, Caltech Brain Imaging Center, Adolphs is the co-author of The neuroscience of emotions in humans and animals (Princeton University Press, forthcoming, 2018).

http://emotion.caltech.edu
\end{abstract}

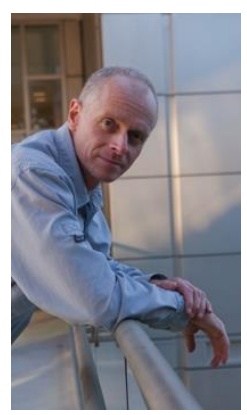

\section{Low specificity and sensitivity.}

Beauchamp (2017) provides a detailed discussion of vigilance, and gives numerous examples from animal behavior. Figure 2 illustrates some of the types of mistakes we can make. Although vigilance is often associated with states of fear, it also plays a role in processing that is not limited to fear, resulting in modest specificity. Beauchamp seems to believe that vigilance in principle offers high sensitivity for detecting fear, but that there are measurement problems that in practice yield low sensitivity. So better measures of vigilance are one important direction for improvement. Another would be to add features that tend to accompany fear in order to improve sensitivity and specificity. Beauchamp considers autonomic and hormonal measures, but concludes that none of them offer particularly reliable markers for fear.

One could think of adding many more dependent measures. Facial expressions, multivariate autonomic measures, and other behavioral reactions have all been explored, but with generally poor specificity and sensitivity, just as Beauchamp notes. It could be that the set of measures is still too small; it could be that the classification approaches used have not been sufficiently sophisticated. But it could also be that such variables in and of themselves can never diagnose fear (or any other emotion) because they only measure responses without considering their functional role, which may need to be evaluated relative to the causes of fear (the situation in which a person or animal finds themselves).

\section{Cause of consequence?}

Everybody seems to agree that fear and vigilance are correlated to some degree. Implicit in 
Beauchamp's article is the possibility of something stronger than mere correlation, but this is also where disagreements will arise. One could include vigilance as constitutive of an extended concept of fear, or as causally related. A natural view is that the causal directionality is from vigilance to fear. Threat imminence theory would seem to endorse such a view (Fanselow \& Lester, 1988). The idea is that vigilance is antecedent to fear in that it is involved in monitoring, attention, mild anxiety, and states that would then gradually evolve into fear - if additional conditions obtain. Under this view, vigilance increases the probability of fear, but is by itself insufficient for fear.

Alternatively, fear could cause vigilance ("reactive vigilance" in Beauchamp's terminology), which surely also obtains under certain conditions. But whether vigilance or particular physiological changes are expressed always needs to be interpreted in light of the adaptive value of the threat situation: fear is the collective change in state variables that mediate an animal's coordinated coping with a threat. Seen in this functional way, the state of fear could actually be constituted (implemented, realized) by different sets of behavioral and physiological changes, depending on the situation and the species under study.

\section{Operationalizing fear.}

One way to think of vigilance, then, is as the implementation of a specific functional component of fear - one whose functional role is to gather information, allocate attention to relevant stimuli and their features, and prepare subsequent routines. In this view, the functions subserved by vigilance may not be appropriate under all circumstances or for all types of fear. It has been suggested that the functional role of vigilance comes into play during specific aspects or stages of fear, such as initial monitoring of a threat, but not others, such as acute defense (e.g., Fanselow \& Lester, 1988; Mobbs et al., 2015). This partitioning or fractionation of a fear state acknowledges the multicomponent nature of emotion states, and suggests that our current categories, like "fear," will likely need to be carved at a finer grain.

One response to these considerations is to conclude that there are many different emotions all subsumed by our current concept of "fear." That is, "fear" actually fractionates into a bunch of different and heterogeneous emotions for dealing with different kinds of threats in different kinds of ways. If this were the case, vigilance may be a reliable indicator only of a subset of these new fear subtypes, or phases of them. If only we make the categories sufficiently fine-grained and precise, vigilance could turn out to be a specific and sensitive indicator of such a fear subtype after all.

Another response is to conclude that "fear" consists of many different functional components, and that vigilance comes into play only for some of these. Depending on the nature of the threat and the temporal phase in processing, vigilance may be assembled as part of the package. But it is possible to retain "fear" as a single emotion category, unified by the global functional role that it plays in how an organism copes with a variety of threats. There is some neuroscience support for this view also: counter to earlier schemes, it now appears that the central amygdala and bed nucleus of the stria terminalis, nuclei in the medial temporal lobe long known to participate in defensive behaviors, form an integrated circuit that can process a large diversity of different types of threat by flexibly recruiting other brain regions (Shackman \& Fox, 2016). 
Either of the above scenarios leaves vigilance as a potentially very useful indicator of fear, once further distinctions have been made (in either defining subtypes of fear, or in defining the components of a unitary fear state). What remains are the problems of measurement that Beauchamp notes prominently in his paper, and the question of where to draw the spatiotemporal boundary for the fear state. With respect to the latter, the unitary fear view may fit better with a rather extended boundary that includes vigilance as one (variably recruited) component that is constitutive of fear. The fear-subtypes view, on the other hand, may fit better with the idea that vigilance is a cause of some, but not all, types of fear.

It is apparent that no single criterion can decide between these multiple schemes. There are functional, psychological, and neurobiological constraints that all need to be optimized in some way. The holistic nature of operationalizing a scientifically useful concept of "fear," and the role of "vigilance" in it, emphasizes the need to include all types of data, and all types of questions. Only through discussions between philosophers, ecologists, psychologists and neurobiologists will we be able to derive a shared scientific concept of fear and vigilance with the greatest utility across disciplines.

\section{References}

Beauchamp, G. (2017). What can vigilance tell us about fear? Animal Sentience 15(1).

Fanselow, M. S., \& Lester, L.S. (1988). A functional behavioristic approach to aversively motivated behavior: Predatory imminence as a determinant of the topography of defensive behavior. In R. C. B. M. D. Beecher (Ed.), Evolution and Learning (pp. 185-211). Hillsdale, N.J.: Erlbaum.

Mobbs, D., Hagan, C., Dalgleish, T., Stilson, B., \& Prevost, C. (2015). The ecology of human fear: Survival optimization and the nervous system. Frontiers in Neuroscience: Evolutionary Psychology and Neuroscience 9: 55.

Shackman, A.J., \& Fox, A.S. (2016). Contributions of the central extended amygdala to fear and anxiety. The Journal of Neuroscience 36: 8050-8063. 